\title{
Implications of the noncrossing property of Bohm trajectories in one-dimensional tunneling configurations
}

\author{
X. Oriols, F. Martín, and J. Suñé \\ Department d'Enginyeria Electrònica, Universitat Autònoma de Barcelona, 08193-Bellaterra, Spain
}

(Received 2 January 1996)

\begin{abstract}
Several practical implications of the noncrossing property of one-dimensional Bohm trajectories are examined. It is shown that the position of a Bohm particle, the average transmission, reflection and dwell times, and the probability distribution of these tunneling times, can all be obtained without actually calculating trajectories. On the other hand, the intuitive interpretation of the scattering of wave packets by potential barriers is discussed within the framework of Bohm's interpretation of quantum mechanics. In this regard, claims that Bohm's approach leads to counterintuitive results are shown to be subjective. [S1050-2947(96)02209-3]
\end{abstract}

PACS number(s): 03.65.-w, 73.40.Gk, 85.30.Mn, 02.70.Lq

\section{INTRODUCTION}

In the context of one-dimensional tunneling configurations, the dwell time is defined as the average (ensemble) time spent by the incoming particles in the potential barrier region $(a<x<b)$ :

$$
\tau_{D}=\int_{0}^{\infty} d t \int_{a}^{b} d x|\Psi(x, t)|^{2} .
$$

This time was first postulated by Büttiker [1], and more recently rigorous derivations have been obtained within Feynman's [2] and Bohm's [3-5] formulations of quantum mechanics (QM). Although there are some divergences regarding its physical interpretation $[6,7]$, this is widely recognized as a meaningful concept $[8,9]$. On the other hand, much more controversy exists about the transmission $\left(\tau_{T}\right)$ and reflection $\left(\tau_{R}\right)$ times, which are sometimes loosely defined as the average times spent in the barrier region by the particles that are ultimately transmitted or reflected, respectively. In the same way, the relation

$$
\tau_{D}=|T|^{2} \tau_{T}+|R|^{2} \tau_{R}
$$

$\left(|T|^{2}\right.$ and $|R|^{2}$ being the transmission and reflection probabilities associated with the wave packet) is also controversial. Some authors have claimed that this is a necessary requirement for any meaningful $\tau_{T}$ and $\tau_{R}$, arguing that transmission and reflection are mutually exclusive events that exhaust all the possibilities [8]. However, it has also been pointed out that the questions, "Will the particle be transmitted" and "Is the particle in the potential barrier region?" correspond to noncommuting observables [9-11]. As a consequence, an additional interference term appears in the right-hand side of Eq. (2) [11]. In our opinion, however, if the definition of the tunneling times given just prior to Eq. (2) is to be interpreted literally, there should be no doubt about the validity of relation (2). Nevertheless, the problem is that this definition is not meaningful within the conventional interpretation of QM because the time spent in the barrier region by one particle cannot actually be measured. To determine this time, two successive measurements of position would be required, but this procedure leads to mean- ingless results due to the collapse of the wave function after the first measurement. For this reason, there is no contradiction if some of the diverse quantities proposed to represent the tunneling times within conventional QM do not satisfy Eq. (2). On the contrary, tunneling times are unambiguously defined within Bohm's interpretation of QM [12-14], which is a causal theory of quantum-mechanical processes in space and time, and not just a theory about experimental results [15]. Since, on the other hand, the Bohm tunneling times perfectly fit the previous general definition, they indeed satisfy Eq. (2), as required [3-5].

According to Bohm's interpretation, an electron is a particle that is guided by a pilot field related to the wave function $\Psi(x, t)$, and follows a well-defined trajectory $x\left(x_{0}, t\right)$, which only depends on its starting position $x_{0}$ within the initial wave packet $\Psi(x, 0)$. A complete description of any quantum-mechanical problem, however, requires the consideration of a (classical) ensemble of trajectories. All measurable quantities, which are obtained by averaging the values of the single trajectories weighted according to $\left|\Psi\left(x_{0}, 0\right)\right|^{2}$, exactly reproduce the results of the standard interpretation of QM. Furthermore, since the particle trajectories are perfectly defined, some quantities that are controversial within the standard interpretation (such as tunneling times) are unambiguous within Bohm's framework. Mainly for this reason, the tunneling of electrons through potential barriers has been recently analyzed by Leavens and Aers within Bohm's interpretation [3-5]. These authors presented the theory in detail, worked out several examples of transmission of wave packets through one-dimensional barriers, obtained the distributions of transmission and reflection times (in addition to their average values), and compared their results with those of other conventional approaches to the tunneling time problem [5]. They also emphasized that one-dimensional Bohm trajectories never intersect at any space-time point, and discussed one of its consequences. There is a bifurcation trajectory (starting at $x_{c 0}$ ) that separates the wave packet in two parts: that to be transmitted $\left(x_{0}>x_{c 0}\right)$, and that to be reflected $\left(x_{0}<x_{c 0}\right)$. This means that, within Bohm's approach, all the transmission comes from the spatial front of the wave packet, and that all the rear part of it is reflected. This had been previously observed by Dewdney and Hiley [16], and motivated the somehow skeptical comments of Landauer and 
Martin [9], who stressed apparent contradictions between these results and the common intuitive interpretation of the scattering of wave packets by potential barriers. In a very recent paper, the noncrossing property of Bohm trajectories was also used by McKinnon and Leavens [17] to obtain the distribution of transmission times without calculating trajectories. Their method is very interesting and significantly reduces the time required to compute this distribution. However, further discussion is required because, as we will show, their procedure cannot be applied to all possible cases.

It is the purpose of this paper to discuss several practical implications of the noncrossing property of Bohm trajectories. In particular, it is shown that the average transmission and reflection times can be obtained without actually calculating a single trajectory, and that the position of a Bohm particle can be directly obtained from the wave function, i.e., without following its trajectory. On the other hand, the method of McKinnon and Leavens [17] is reformulated so that it can be used to obtain the distributions of transmission, reflection, and dwell times corresponding to arbitrary wave packets and potential barriers, without calculating trajectories. Finally, subjective implications of the fact that Bohm trajectories never intersect are also discussed. In this regard, we try to reconcile the results obtained within Bohm's approach with the common sense interpretation of the scattering processes in one-dimensional tunneling configurations. We center our discussions in double-barrier resonant tunneling structures (DBRTS) because the phenomenology is richer than in the simpler case of single barriers. In all the numerical examples, physical parameters (effective mass, barrier heights and thicknesses, etc.) typical of the GaAs/ $\mathrm{AlGaAs}$ system are considered.

\section{NONCROSSING PROPERTY OF BOHM TRAJECTORIES}

In Bohm's interpretation of nonrelativistic QM [12-14], an electron is a particle the motion of which is completely determined by an objectively real field related to the wave function $\Psi(x, t)$, so that it has a well-determined position and velocity at each instant of time, i.e., a well-defined trajectory. In this casual interpretation, $\Psi(x, t)$ is a solution of the time-dependent Schrödinger equation (TDSE), the velocity at any space-time point is uniquely given by $v(x, t)$ $=\partial S(x, t) / \partial x[S(x, t)$ being the phase of the complex wave function], and $|\Psi(x, t)|^{2} d x$ is the probability of the electron being between $x$ and $x+d x$ at time $t$ even in the absence of a position measurement. The description of any scattering problem requires the choice of an initial wave function $\Psi(x, 0)$ adequate to the particular situation, and the analysis of its subsequent time evolution. A description in terms of scattering energy eigenstates is not convenient because these are stationary states and time evolution is inherent to the concept of trajectory. Moreover, if stationary states are used, unphysical and inconsistent results come out within Bohm's framework $[18,19]$. In any case, this is not a limitation of Bohm's interpretation but rather a consequence of the nature of these states themselves which, not being normalized, cannot be a perfect description of the quantum system. For these reasons, localized time-dependent wave packets have to be used to analyze scattering processes within Bohm's ap- proach. Assuming a particular $\Psi(x, 0)$, and given the position $x_{0}$ of an electron within the wave packet, its subsequent trajectory $x\left(x_{0}, t\right)$ is uniquely determined by simultaneous integration of the TDSE and the guidance equation $d x / d t$ $=v(x, t)$. However, due to the uncertainty principle, the position of the electron at $t=0$ cannot be precisely known and, as a consequence, one must deal with an ensemble of trajectories that can be labeled by $x_{0}$. To determine the expectation value of any function (observable or not) one has to average the results of all the possible trajectories according to a weight given by $\left|\Psi\left(x_{0}, 0\right)\right|^{2} d x$.

The Bohm trajectories can also be viewed as the solutions of a modified Hamilton-Jacobi equation [12] and, as such, they cannot cross each other in the configuration space. Moreover, since the velocity of the Bohm particles is uniquely determined by $\partial S(x, t) / \partial x$, it immediately follows that the trajectories do not cross in space-time either. In fact, if two trajectories should cross at a point $(x, t)$, the corresponding velocities would also be identical, and the trajectories would cross in the configuration space. In onedimensional systems, the fact that Bohm trajectories do not intersect each other means that any pair of particles starting at initial points $x_{02}>x_{01}$ will maintain their relative positions all the time, i.e., $x\left(x_{02}, t\right)>x\left(x_{01}, t\right)$. Since, on the other hand, the probability density $|\Psi(x, t)|^{2}$ is directly related to the positions of the Bohm particles,

$$
|\Psi(x, t)|^{2}=\int_{-\infty}^{\infty} d x_{0}\left|\Psi\left(x_{0}, 0\right)\right|^{2} \delta\left(x-x\left(x_{0}, t\right)\right),
$$

it follows that the total probability presence at the right (or left) of any trajectory is constant for all the times. Thus, if we define $Q(x, t)$ as the probability presence to the right of point $x$ at time $t$ :

$$
Q(x, t) \equiv \int_{x}^{\infty}\left|\Psi\left(x^{\prime}, t\right)\right|^{2} d x^{\prime}=\int_{0}^{t} J\left(x, t^{\prime}\right) d t^{\prime},
$$

we can also label each trajectory by $Q\left(x_{0}\right) \equiv Q\left(x_{0}, 0\right)$ and the probability presence to the right of $x\left(x_{0}, t\right)$ is always $Q\left(x_{0}\right)$ :

$$
Q\left(x_{0}\right) \equiv \int_{x_{0}}^{\infty} d x|\Psi(x, 0)|^{2}=\int_{x\left(x_{0}, t\right)}^{\infty} d x|\Psi(x, t)|^{2}
$$

The first consequence of the noncrossing property of Bohm trajectories, already pointed out by Leavens and Aers [5], is that there is a bifurcation trajectory $x_{c}(t)=x\left(x_{c 0}, t\right)$ implicitly given by

$$
|T|^{2}=\int_{x_{c}(t)}^{\infty} d x|\Psi(x, t)|^{2}
$$

which divides the wave packet in two spatially separated parts, $\left|\Psi_{T}(x, t)\right|^{2}$ and $\left|\Psi_{R}(x, t)\right|^{2}$, which are to be transmitted and reflected, respectively:

$$
\begin{aligned}
& \left|\Psi_{T}(x, t)\right|^{2}=|\Psi(x, t)|^{2} \Theta\left[x-x_{c}(t)\right], \\
& \left|\Psi_{R}(x, t)\right|^{2}=|\Psi(x, t)|^{2} \Theta\left[x_{c}(t)-x\right],
\end{aligned}
$$

$\Theta[x]$ being the unit step Heaviside function. This is particlelike decomposition [20], which allows the calculation of the 
average transmission and reflection times directly from the wave function without weighting trajectories [5]:

$$
\begin{gathered}
\tau_{T}=\frac{1}{|T|^{2}} \int_{0}^{\infty} d t \int_{a}^{b} d x\left|\Psi_{T}(x, t)\right|^{2}, \\
\tau_{R}=\frac{1}{|R|^{2}} \int_{0}^{\infty} d t \int_{a}^{b} d x\left|\Psi_{R}(x, t)\right|^{2} .
\end{gathered}
$$

Notice that only the bifurcation trajectory $x_{c}(t)$ is needed to obtain $\left|\Psi_{T}(x, t)\right|^{2}$ and $\left|\Psi_{R}(x, t)\right|^{2}$, and that this directly allows the calculation of $\tau_{T}$ and $\tau_{R}$. However, the explicit calculation of $x_{c}(t)$ is not required to obtained these tunneling times, as it will be shown below.

The noncrossing property of Bohm trajectories has other interesting practical consequences that were not discussed in [5]: (1) the position of any Bohm particle can be obtained at any arbitrary time without calculating the corresponding trajectory; and (2) the causal distributions of transmission, reflection, and dwell times can also be directly determined from the time-evolved wave function. Section III is entirely dedicated to show how these distributions can be obtained without evaluating trajectories, and the rest of this section is devoted to the discussion of how to assess the position of any Bohm particle from $\Psi(x, t)$, and to some practical implications of this property. From Eq. (5), it immediately follows that we can determine the position of a Bohm particle at any time by integrating the presence probability. In other words, for a particle starting at $x_{0}$, we can compute its position $x\left(x_{0}, t_{0}\right)$ at any arbitrary instant of time $t_{0}$ without wondering about its trajectory between $t=0$ and $t=t_{0}$. In fact, having calculated $Q\left(x_{0}\right)$ from the initial wave packet, we can determine $x\left(x_{0}, t_{0}\right)$ by spatial integration of the time-evolved wave function $\Psi\left(x, t_{0}\right)$. This is quite a trivial result, but it may have very interesting practical consequences. If, for example, Bohm trajectories were used for the extension of the Monte Carlo (MC) simulation technique to quantum-based electron devices such as resonant tunneling diodes [21], the direct calculation of the position of the Bohm particles would significantly reduce the computation times. In a MC scheme, the time of flight is chosen through the generation of a random number according to the total scattering rate. Using Eq. (5), we would be able to obtain the position of the electrons after their free flight without computing their entire trajectory. This would largely improve the numerical efficiency because of the huge number of trajectories that should otherwise be calculated. Let us finally indicate that the convenience of this procedure to assess the position of the Bohm particles can be enhanced by the use of an appropriate method to solve the TDSE. In this regard, instead of timediscretizing this equation, as it is usually done [22], it is more convenient to begin by numerically solving the stationary Schrödinger equation (see, for example, [23,24]), and then project the initial wave packet onto the basis of Hamiltonian scattering eigenstates $\Psi_{E}(x)$. In this way, the timedependent wave function can be directly obtained by superposition, i.e., without calculating it at intermediate times:

$$
\Psi\left(x, t_{0}\right)=\int_{0}^{\infty} a(E) e^{-i\left(E t_{0}\right) / \hbar} \Psi_{E}(x) d E
$$

with $a(E)=\int_{0}^{\infty} \Psi_{E}^{*}(x) \Psi(x, 0) d x$. Thus, to obtain the position of any Bohm particle at time $t_{0}$, we do not need to evaluate the associated wave function nor its trajectory at intermediate times between $t=0$ and $t=t_{0}$. This method, which is the one used in this paper to integrate the TDSE is only valid for time-independent potential profiles (this is the case between successive actualizations of the potential in a MC simulation), and has the additional advantage of avoiding spurious reflections at the boundaries of the integration box [22].

\section{DISTRIBUTION OF TUNNELING TIMES}

As we have advanced in Sec. II, another important consequence of the noncrossing property of Bohm trajectories is that the distributions of transmission, reflection, and dwell times can also be be obtained without calculating trajectories. As for the transmission time distribution, McKinnon and Leavens have recently discussed how to evaluate it by connecting the arrival time distributions at the two boundaries of the barrier $(x=a$ and $x=b)$ with the help of Eq. (5) [17]. Although we agree with their basic idea of connecting arrival time distributions, we want to point out that their method is not completely general. In particular, they implicitly assumed that different Bohm particles must have different transmission times and, although this is the case in the most common situations, this is not true in general. This is discussed in detail below, and a reformulation of McKinnon and Leavens's method is presented to overcome its limitations.

Recently, Muga, Brouard, and Macías have rigorously justified the use of the current density as an arrival time distribution within the conventional interpretation of $\mathrm{QM}$ [25]. If $J(x, t)$ is the probability current density at point $x$ and time $t$, the quantum-mechanical particles cross (arrive at) this point distributed according to

$$
P_{x}(t)=\frac{|J(x, t)|}{\int_{-\infty}^{\infty}|J(x, t)| d t} .
$$

The absolute value is required to take into account that the current density can take negative values, and the normalization is needed because it is neither guaranteed that all the particles arrive at point $x$, nor that they do not cross the $x$ interface several times from left to right and vice versa. Equation (10) is quite intuitive, and had already been previously proposed by other authors [10,26,27]. Following McKinnon and Leavens [17], we consider $Q(x, t)$ [see Eq. (4)] at the boundaries of the barrier, i.e., $Q(a, t)$ and $Q(b, t)$. For the typical problem of a Gaussian wave packet impinging upon a DBRTS (see diagram in Fig. 1) from left to right, $Q(a, t)$ and $Q(b, t)$ appear as shown in Fig. 2. Notice that $Q(b, t)$ increases monotonously towards $|T|^{2}$ (though oscillating for reasons that will be discussed below), and that this means that $J(b, t)>0$ at all times. In this regard, we have to point out that although the positivity of the current density has been recently demonstrated for the asymptotic region sufficiently far from the barrier [25], it can be shown that the current density can eventually take negative values at $x=b$ in extreme situations (capricious wave packets). However, in the rest of the paper we will assume that $J\left(b, t_{b}\right)>0$ or, in other words, we will neglect the possibility of reentrant 


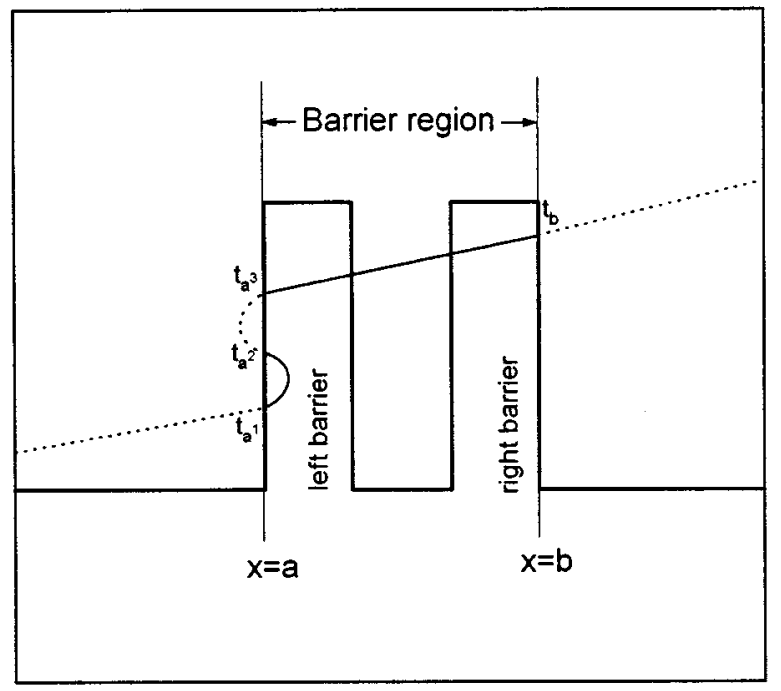

FIG. 1. Schematic diagram of the considered symmetric doublebarrier heteroestructures. A hypothetical trajectory is used to define $t_{a}\left(t_{a}^{i}\right)$ and $t_{b}$ as the times the trajectory crosses the $x=a$ and the $x=b$ interface, respectively.

Bohm trajectories at the $x=b$ interface. In this sense, our numerical results of this and previous works have always confirmed this assumption $[19,28]$, in spite of the fact that in QM the probability current can take negative values even for states having only positive momentum components [29]. This is in agreement wiht Leavens and Aers's results [5], who never found Bohm trajectories crossing the $x=b$ boundary from right to left. In Leavens's words, "a flag in the

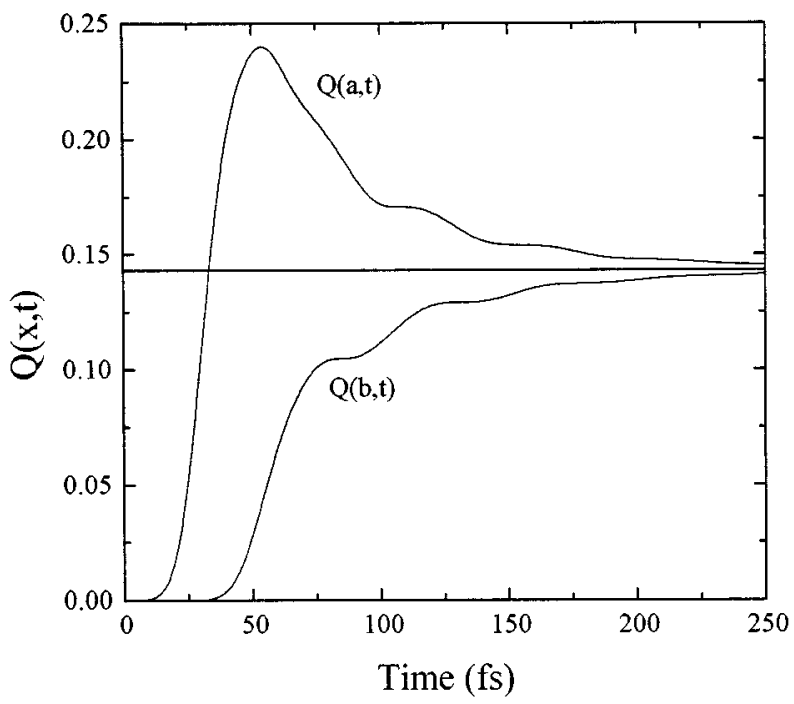

FIG. 2. Probability presence at the right of the extreme doublebarrier points $Q(a, t)$ and $Q(b, t)$ as a function of time. These results correspond to the resonant transmission of a Gaussian electron wave packet (with the effective mass of point $\Gamma$ in GaAs, i.e., 0.067 times the free-electron mass) incident upon a double-barrier potential typical of the GaAs/AlGaAs system: barrier height of $0.3 \mathrm{eV}$, barrier thickness $2 \mathrm{~nm}$, and well width of $18 \mathrm{~nm}$. The well is wide so as to observe the oscillations in the Bohm trajectories. The horizontal line corresponds to $Q(t)=|T|^{2}$, or in other words, to the $x_{c}(t)$ trajectory. author's computer codes to monitor reentrant trajectories at $x=b$ has yet to be triggered"' [27]. This is not the case, of course, at the $x=a$ boundary. In particular, all the reflected particles that cross this interface from left to right cross it again from right to left. For the case shown in Fig. 2, $Q(a, t)$ increases monotonously [though oscillating as $Q(b, t)$ ] before reaching its maximum and then it decreases towards $|T|^{2}$. However, this behavior is not the most general one (as it will be shown below) because, depending on the shape of the initial wave packet, $Q(a, t)$ can present several relative maxima, which correspond to particles that cross the $x=a$ boundary more than once before being finally reflected or transmitted.

\section{A. Average tunneling times}

Before discussing the method to calculate the tunneling time distributions, let us show how $\tau_{D}, \tau_{T}$, and $\tau_{R}$ can all be obtained from $Q(a, t)$ and $Q(b, t)$, i.e., without evaluating a single trajectory. For the sake of simplicity, we begin by considering cases as that of Fig. 2, i.e., with $Q(a, t)$ showing a single maximum. First of all, notice that the probability presence in the barrier region, $D(t)=\int_{a}^{b}|\Psi(x, t)|^{2} d x$, is equal to $Q(a, t)-Q(b, t)$. Thus, according to Eq. (1), the dwell time is

$$
\tau_{D}=\int_{0}^{\infty}[Q(a, t)-Q(b, t)] d t
$$

and this corresponds to the area enclosed by the two curves of Fig. 2. It can also be shown that this area is divided by the horizontal line $Q(t)=|T|^{2}$ in two subareas that are proportional to $\tau_{T}$ and $\tau_{R}$, respectively. Effectively, since Bohm trajectories do not cross, all the particles that have entered the barrier are to be finally transmitted if $Q(a, t)<|T|^{2}$. Thus, for $t<t_{1}$ [with $t_{1}$ implicitly defined by $Q\left(a, t_{1}\right)=|T|^{2}$ ], the probability presence of to be transmitted particles in the barrier region, $D_{T}(t)=\int_{a}^{b}|\Psi(x, t)|^{2} d x$, is equal to $Q(a, t)$ $-Q(b, t)$, and coincides with $D(t)$. For $t>t_{1}$, only those particles located at the right of $x_{c}(t)$ (which for $t>t_{1}$ is located within the barrier or at the right side of it) are to be transmitted, and hence $D_{T}(t)=|T|^{2}-Q(b, t)$. As a consequence, the average transmission time is given by

$$
\begin{aligned}
\tau_{T}= & \frac{1}{|T|^{2}} \int_{0}^{t_{1}}[Q(a, t)-Q(b, t)] d t \\
& +\frac{1}{|T|^{2}} \int_{t_{1}}^{\infty}\left[|T|^{2}-Q(b, t)\right] d t .
\end{aligned}
$$

This demonstrates that the area enclosed by the two curves of Fig. 2, $Q(a, t), Q(b, t)$, and by the horizontal line $Q(t)=|T|^{2}$, is equal to $|T|^{2} \tau_{T}$. On the other hand, from Eq. (2) it follows that the rest of the area enclosed by $Q(a, t)$ and $Q(b, t)$, i.e., the area over $Q(t)=|T|^{2}$ and below $Q(a, t)$, is equal to $|R|^{2} \tau_{R}$. This can also be formulated as

$$
\tau_{R}=\frac{1}{|R|^{2}} \int_{t_{1}}^{\infty}\left[Q(a, t)-|T|^{2}\right] d t .
$$

In the most general case when the wave packet is such that the corresponding $Q(a, t)$ has several maxima and crosses 
the $Q(t)=|T|^{2}$ line several times before decreasing towards $|T|^{2}$, the procedure to evaluate the average tunneling times is analogous. The case of the dwell time is trivial because $\tau_{D}$ is always given by Eq. (11), independently of the shape of $Q(a, t)$. The other two times can also be obtained from $Q(a, t)$ and $Q(b, t)$, but this requires a straightforward generalization of Eqs. (12) and (13). In the most general case, it can be easily demonstrated that the transmission time is given by

$$
\tau_{T}=\frac{1}{|T|^{2}} \int_{0}^{\infty}\left\{\min \left[Q(a, t),|T|^{2}\right]-Q(b, t)\right\} d t
$$

and the reflection time by

$$
\tau_{R}=\frac{1}{|R|^{2}} \int_{0}^{\infty}\left\{\max \left[Q(a, t),|T|^{2}\right]-|T|^{2}\right\} d t,
$$

Eqs. (12) and (13) being particular cases of Eqs. (14) and (15), respectively. This demonstrates that the calculation of trajectories is not needed to obtain the average tunneling times. In particular, the calculation of $x_{c}(t)$ is also not required, since the decomposition of the wave packet into to be transmitted and to be reflected components is implicitly done when the area between $Q(a, t)$ and $Q(b, t)$ is divided by the horizontal line $Q(t)=|T|^{2}$. In this regard, notice that in a $Q=Q(t)$ plot, the Bohm trajectories fall on horizontal lines because of their noncrossing property, and that $Q(t)=|T|^{2}$ actually corresponds to the bifurcation trajectory $x_{c}(t)$.

\section{B. Transmission time distribution}

As discussed by McKinnon and Leavens [17], the problem of calculating the transmission time distribution can be reduced to matching points of the arrival distribution at $x=a$ with points of the arrival distribution at $x=b$. In the standard interpretation of QM, this matching is not possible because the actual concept of a particle sequentially arriving at two points is meaningless. On the contrary, within Bohm's approach, there is a well-defined procedure that consists in identifying which particle arrives at these boundaries at each instant of time. In this regard, we have seen that a Bohm particle (trajectory) can be labeled by its starting position $x_{0}$. However, the noncrossing property of the trajectories provides an alternative identification method, which consists in labeling the particles by their corresponding value of $Q\left(x_{0}\right)$, as defined in Eq. (5). In this way, the particle that arrives at the $x=b$ boundary at $t=t_{b}$ is determined by $Q\left[x_{0}\left(t_{b}\right)\right]$ $=Q\left(b, t_{b}\right)$, and one can readily calculate the instant of time $t_{a}$ at which the same particle crossed $x=a$ by requiring $Q\left(a, t_{a}\right)=Q\left[x_{0}\left(t_{b}\right)\right]$. This latter equation can have more than one solution because the particles can cross the $x=a$ interface several times. However, and for the sake of simplicity, we will first consider that (as in Fig. 2) the transmitted particles only cross this boundary once. In this simplest case, the transmission time of the particle which starts at $x_{0}\left(t_{b}\right)$ is just $t_{a b}=t_{b}-t_{a}$.

Provided that there are no reentrant trajectories at $x=b$, the distribution of arrival times at this interface can be calculated from $Q(b, t)$ as

$$
P_{b}\left(t_{b}\right)=\left.\frac{1}{|T|^{2}} \frac{\partial Q(b, t)}{\partial t}\right|_{t=t_{b}} .
$$

For this reason, McKinnon and Leavens [17] identified $Q\left(b, t_{b}\right)$ as $|T|^{2}$ times the cumulative arrival time distribution function associated with $P_{b}\left(t_{b}\right)$. After that, they considered that the cumulative transmission time distribution $Q_{T}\left(t_{a b}\right)$ could be calculated by inverting the relation

$$
t_{a b}\left(Q\left(x_{0}\right)\right)=t_{b}\left(Q\left(x_{0}\right)\right)-t_{a}\left(Q\left(x_{0}\right)\right),
$$

i.e., by determining the value of $Q\left(x_{0}\right)$ that corresponds to each transmission time $t_{a b}$, and assuming that $Q_{T}\left(t_{a b}\right)$ $=Q\left(x_{0}\right)$. Finally, they evaluated the transmission time distribution $P_{T}\left(t_{a b}\right)$ by differentiating $Q_{T}\left(t_{a b}\right)$ with respect to $t_{a b}$ and normalizing by $|T|^{2}$. However, this procedure is correct only if all the transmitted particles have different transmitted times, i.e., if the relation between $t_{a b}$ and $Q\left(x_{0}\right)$ is single valued. This condition is satisfied for all the cases worked out in [17] but, in the most general case, several particles can have the same transmission time and, as a consequence, Eq. (17) cannot be inverted. This will be explicitly shown by means of an example but, for the moment, let us reformulate the method so that it can be applied to all possible cases.

To calculate the transmission time distribution, we propose to proceed as follows: first that the transmission time $t_{a b}$ is calculated for each value of $t_{b}$, i.e., for all the Bohm particles that arrive at $x=b$. In this way, a function $t_{a b}=t_{a b}\left(t_{b}\right)$ is obtained. If the transmission time is different for all the trajectories, i.e., if the function $t_{a b}\left(t_{b}\right)$ is monotonic, the transmission time distribution $P_{T}\left(t_{a b}\right)$ is directly given by

$$
P_{T}\left(t_{a b}\right)=P_{b}\left(t_{b}\right)\left[\frac{d t_{a b}\left(t_{b}\right)}{d t_{b}}\right]^{-1}=\frac{1}{|T|^{2}} J\left(b, t_{b}\right)\left[\frac{d t_{a b}\left(t_{b}\right)}{d t_{b}}\right]^{-1} .
$$

This expression is exactly equivalent to the inversion of Eq. (17) proposed by McKinnon and Leavens [17], and it reveals that the transmission distribution is obtained from a local renormalization of the arrival time distribution. For the particular case of Fig. 2, which corresponds to a Gaussian wave packet impinging upon a DBRTS, the function $t_{a b}\left(t_{b}\right)$ is shown in Fig. 3(a). After a certain time delay, the relation between $t_{a b}$ and $t_{b}$ becomes linear and with unity slope, and this occurs when all the to be transmitted particles have crossed the $x=a$ interface. Since $t_{a b}$ increases monotonously with $t_{b}$, Eq. (18) can be used to calculate $P_{T}\left(t_{a b}\right)$, as it is explicitly demonstrated in Fig. 3(b), where the obtained distribution is compared to that evaluated by integration of $2.5 \times 10^{4}$ Bohm trajectories. The periodic bumps of the obtained distribution are due to the presence of Bohm trajectories that oscillate in the well of the DBRTS before being finally transmitted, and which are also the cause for the oscillatory structure in $Q(a, t)$ and $Q(b, t)$ of Fig. 2. Equation (18) is valid for a monotonic $t_{a b}\left(t_{b}\right)$ but, as previously said, this function can be nonmonotonic under some circumstances. In these situations, several discrete values $N\left(t_{a b}\right)$ of arrival times, $t_{b}^{i}$ [with $1<i<N\left(t_{a b}\right)$ ], give exactly the same transmission time $t_{a b}$. The dependence of $N$ on $t_{a b}$ is explic- 

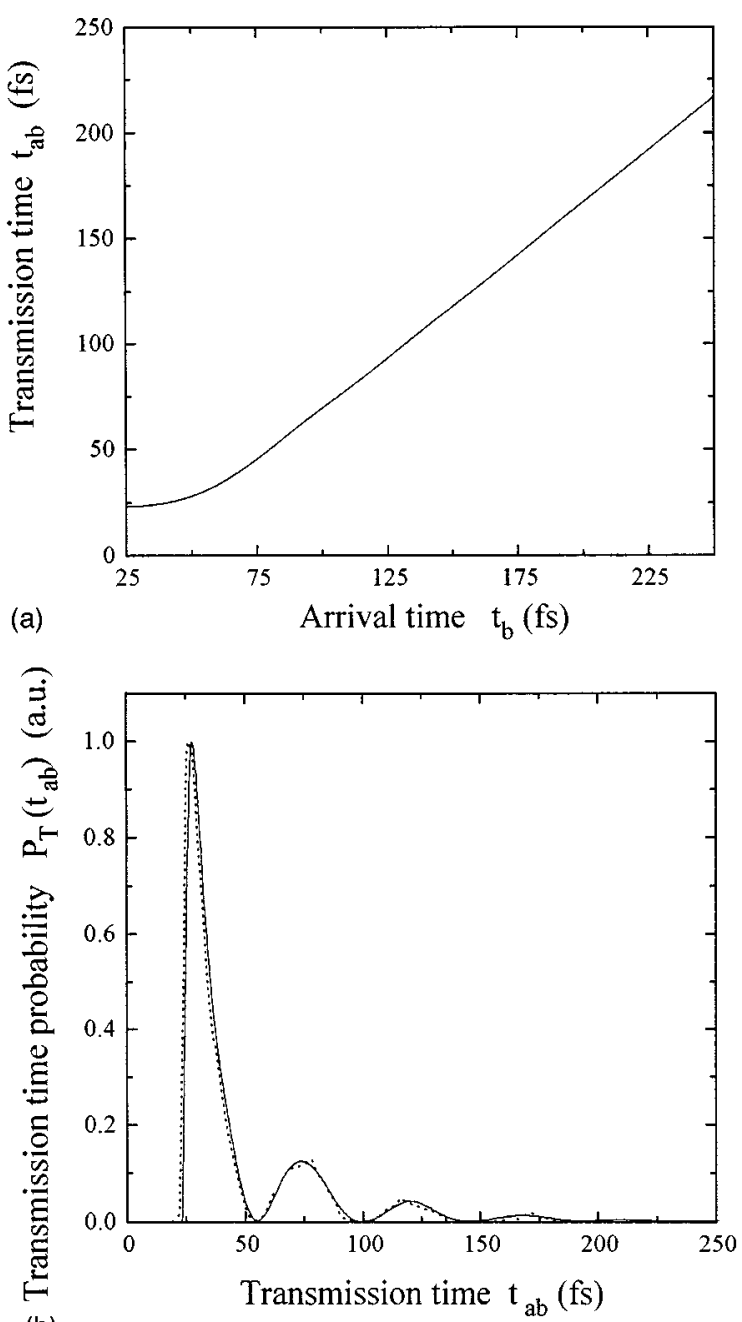

(b)

FIG. 3. (a) Transmission time as a function of time of arrival at the $x=b$ interface for the scattering process of Fig. 2. (b) Transmission time distribution obtained by calculating $2.5 \times 10^{4}$ trajectories (dotted line) compared with that obtained using the procedure presented in the text (continuous line).

itly indicated to emphasize that the number of Bohm particles that have identical transmission times can be different for the different $t_{a b}$ values. In any case, since $t_{a b}\left(t_{b}\right)$ is not single valued, Eq. (17) cannot be inverted to obtain $P_{T}\left(t_{a b}\right)$ as proposed by McKinnon and Leavens [17]. However, the generalization of Eq. (18) is straightforward, as it only requires to sum up the contribution of all the $N\left(t_{a b}\right)$ particles. In this way, the transmission time distribution can be calculated as

$$
P_{T}\left(t_{a b}\right)=\sum_{i=1}^{N\left(t_{a b}\right)} \frac{1}{|T|^{2}} J\left(b, t_{b}^{i}\right)\left[\left.\frac{d t_{a b}\left(t_{b}\right)}{d t_{b}}\right|_{t_{b}=t_{b}^{i}}\right]^{-1} .
$$

Before considering a particular example of this situation, let us remove the assumption that the left boundary of the barrier is only crossed once by each transmitted trajectory. Even in this case, however, if the particle is to be transmitted, we know that the $x=a$ boundary has to be crossed an odd number of times, $N^{\prime}\left(t_{a b}\right)$. These multiple crossings affect the calculation of $t_{a b}$ since the equation $Q\left(a, t_{a}\right)=Q\left[x_{0}\left(t_{b}\right)\right]$ has
$N^{\prime}\left(t_{a b}\right)$ solutions $t_{a}^{i}$ that correspond to the sequential arrival of the $x_{0}\left(t_{b}\right)$ particle to the point $x=a$ from left to right and vice versa. When a particle crosses $x=a$ from left to right, it enters the barrier region, and when the crossing takes place in the opposite sense, the particle abandons the barrier. Only those time intervals with the particle within the barrier have to be counted up to calculate the transmission time to be consistent with the definition of $\tau_{T}$ as the average time spent by the transmitted particles in the barrier region. In other words, we have to determine the time elapsed from the very first crossing of $x=a$ (i.e., $t_{a}^{1}$ ) to the time when it finally crosses $x=b$ (i.e., $t_{b}$ ), but we have to subtract the time intervals between right-to-left and left-to-right crossings. Thus, the transmission time is given by

$$
t_{a b}=t_{b}-t_{a}^{1}-\sum_{n=1}^{\left[N^{\prime}\left(t_{a b}\right)-1\right] / 2}\left(t_{a}^{2 n+1}-t_{a}^{2 n}\right)
$$

when the $x=a$ boundary is crossed $N^{\prime}\left(t_{a b}\right)$ times by the same particle. The rest of the procedure to calculate the transmission time distribution is identical to that corresponding to a monotonic $Q(a, t)$. As an example of the need of Eqs. (20) and (19) to calculate the transmission time and the transmission time distribution, respectively, we consider a wave function composed of two spatially separated Gaussian wave packets impinging upon a DBRTS. A similar example, initially studied by Leavens and Aers [5], was subsequently used by Landauer and Martin to comment on the counterintuitive consequences of the noncrossing property of Bohm trajectories [9]. Section IV is entirely devoted to the intuitive interpretation of scattering events within Bohm's approach, and this example will be further examined. However, let us now concentrate on how the transmission time distribution can be calculated in this case. The electrons are considered to be prepared at $t=0$ in the wave function:

$$
\begin{aligned}
\Psi(x, 0)= & \frac{1}{\Omega\left(\pi \sigma_{1}^{2}\right)^{1 / 4}} \exp \left(i k_{1} x\right) \exp \left[-\frac{\left(x-x_{1}\right)^{2}}{2 \sigma_{1}^{2}}\right] \\
& +\frac{1}{\Omega\left(\pi \sigma_{2}^{2}\right)^{1 / 4}} \exp \left(i k_{2} x\right) \exp \left[-\frac{\left(x-x_{2}\right)^{2}}{2 \sigma_{2}^{2}}\right],
\end{aligned}
$$

which is composed of two packets centered at the coordinate points $x_{1}$ and $x_{2}$ (with $x_{1}<x_{2}$ ), and at wave numbers $k_{1}$ and $k_{2}$ (with $k_{1}<k_{2}$ ), and which evolves towards a DBRTS that has a transmission resonance at $k_{1}$. The constant $\Omega$ is for normalization, and $\sigma_{1}$ and $\sigma_{2}$ are the standard deviations of two successive packets. The actual parameters that define the barrier and the wave function are those specified in the caption of Fig. 4. This figure shows the corresponding cumulative arrival time distributions at $x=a$ and $x=b$, i.e., $Q(a, t)$ and $Q(b, t)$, respectively. The asymptotic behavior at $t \rightarrow \infty$ is identical to that shown in Fig. 2, i.e., both $Q(a, t)$ and $Q(b, t)$ converge towards $|T|^{2}$ because the probability presence at the right side of the barrier is equal to $|T|^{2}$ when the scattering event is finished. As expected, $Q(b, t)$ increases monotonously because of the positivity of $J(b, t)$. On the other hand, however, the behavior of $Q(a, t)$ is different from that shown in Fig. 2 in several respects. First of all, 


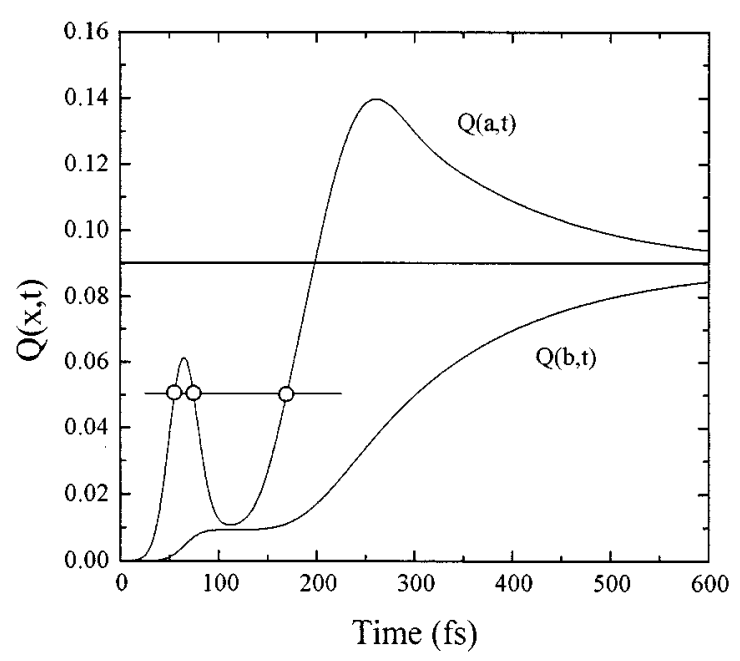

FIG. 4. Probability presence at the right of the extreme barrier points $Q(a, t)$ and $Q(b, t)$ as a function of time. These results correspond to the double Gaussian wave packet $\left(m^{*}=0.067 m_{0}\right)$ described in the text [Eq. (21)] impinging upon a double-barrier potential with $0.3-\mathrm{eV}$-barrier height, 3-nm-thick barriers, and 5-nmwide well. The horizontal corresponds to $Q(t)=|T|^{2}$, i.e., it separates transmitted and reflected particles. The horizontal line (whose crossing are marked with open circles) corresponds to one of the Bohm particles that cross the $x=a$ interface three times before being finally transmitted.

notice that $Q(a, t)$ shows two relative maxima before finally decreasing towards $|T|^{2}$. These maxima are related to the arrival and reflection of the two successive packets but, as we will later show explicitly, only particles from the first one enter into the barrier region in the two successive attempts to cross it. When $Q(a, t)$ decreases after the first relative maximum, some of the particles that have entered the barrier are provisionally reflected (i.e., they cross the $x=a$ interface from right to left) before being thrown again towards the barrier after a collision with the second wave packet. Since the magnitude of the first maximum is smaller than $|T|^{2}$, all the particles contributing to it are to be finally transmitted. As a consequence, some of the transmitted particles cross the $x=a$ interface three times (see the horizontal line and the circles in Fig. 4). For these particles, Eq. (20) is required to calculate their transmission time because during the interval between the second and the third crossings they are outside the barrier region. On the other hand, if we look in Fig. 5(a) at the relation $t_{a b}\left(t_{b}\right)$ obtained using this equation, we perceive that it contains negative slope regions. These negative slopes are related to the extrema of $Q(a, t)$ and, in particular, to the first minimum and the first maximum, respectively. It must be said that several crossings of the $x=a$ interface are a necessary (though not sufficient) condition for having a nonmonotonic relation between $t_{a b}$ and $t_{b}$. On the other hand, this nonmonotonic behavior means that several Bohm particles cross the barrier in exactly the same transmission time. In particular, as shown in the inset of Fig. 5(a), in the particular example that we are analyzing, there are groups of three particles with identical transmission times. In cases as this one, Eq. (17) cannot be inverted, and the calculation of $P_{T}\left(t_{a b}\right)$ requires the use of our reformulated procedure that is explicitly represented by Eq. (19). Using this procedure,
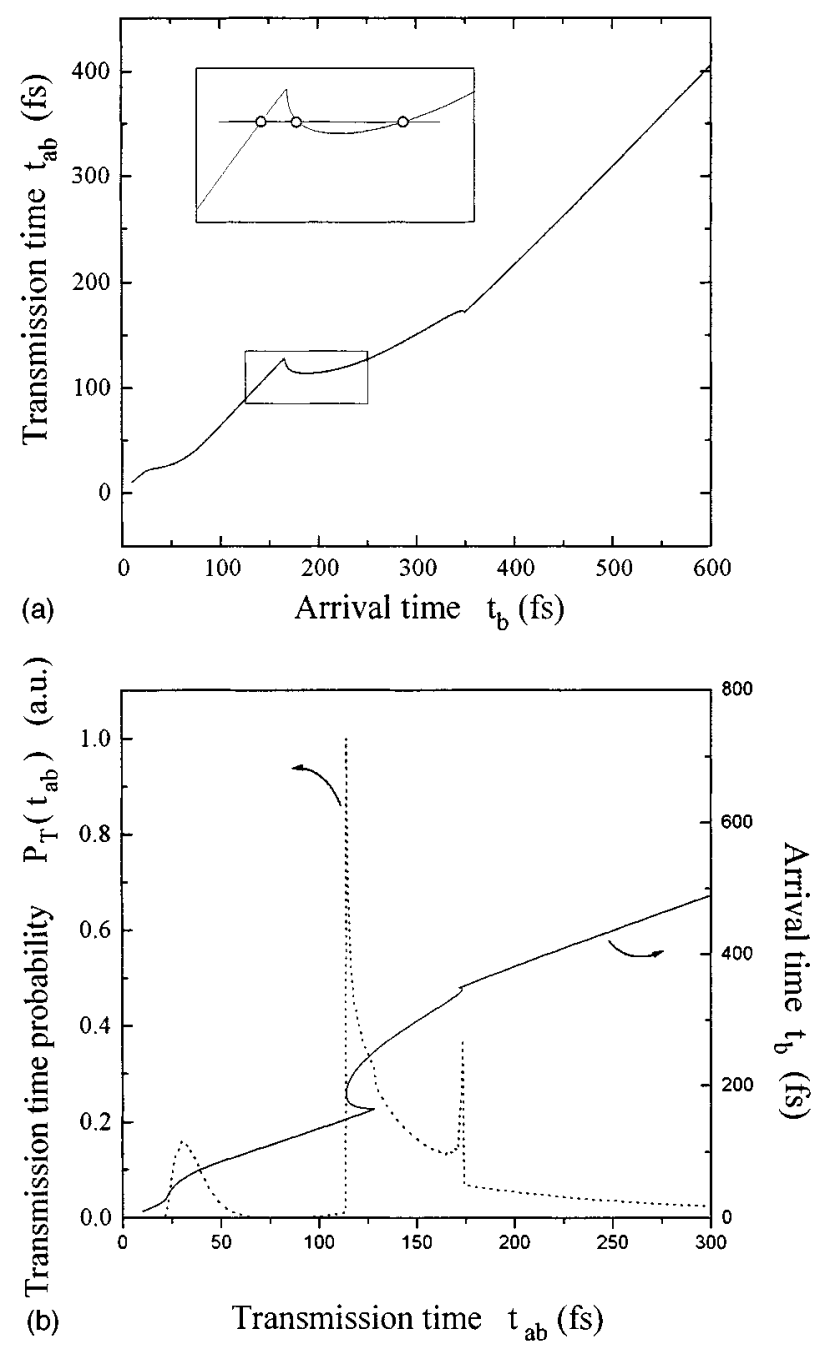

FIG. 5. (a) Transmission time versus arrival time at the $x=b$ interface for the scattering process of Fig. 4. The inset highlights a region with negative slope and the fact that some groups of three Bohm particles (indicated by open circles) that arrive at $x=b$ at different times have exactly the same transmission time. (b) Transmission time distribution associated with the scattering of the double-packet wave function. The $t_{a b}\left(t_{b}\right)$ curve of (a) is repeated here to explain the origin of the two sharp peaks of the transmission distribution.

the transmission time distribution shown in Fig. 5(b) has been obtained without calculating a single trajectory. The curve $t_{a b}\left(t_{b}\right)$ of Fig. 5(a) has also been repeated in Fig. 5(b) to enlighten some relevant features of the transmission time distribution. The first broad bump of this distribution is related to the particles that are transmitted during the first interaction of the front wave packet with the barrier. This can be appreciated in Fig. 4, where $Q(b, t)$ is seen to increase and saturate for $t_{b}<100 \mathrm{fs}$, showing a flat terrace that extends up to $t_{b}>150 \mathrm{fs}$, and which corresponds to the time interval elapsed between the two successive interactions of the front wave packet with the barrier (these sequential interactions and the fact that all the transmission comes from the front packet will become more evident in Sec. IV). Returning back to the description of the transmission distribution of Fig. 5(b), we appreciate a second broad peak that corresponds to the second interaction of the packet with the 
barrier. This second bump, however, is limited by two sharp peaks that can be understood by looking at the superposed $t_{a b}\left(t_{b}\right)$ function. In this regard, we must notice that eventual flat regions in the $t_{a b}\left(t_{b}\right)$ characteristic are an indication of particles that arrive at different instants of time to $x=b$ and that spend exactly the same time in the barrier region. These flat regions would consequently lead to $\delta$ functions in the transmission time distribution. In the same way, the extrema of $t_{a b}\left(t_{b}\right)$ are locally flat regions which lead to sharp peaks [as those of Fig. 5(b)] in the transmission time distribution because $d t_{a b} / d t_{b}=0$.

\section{Distribution of dwell and reflection times}

Although we have used the arrival time distribution at $x=b$ as the starting point to calculate $P_{T}\left(t_{a b}\right)$, an analogous procedure can be followed using the arrival distribution at $x=a$. In this regard, it is straightforward to demonstrate that the transmission time distribution can also be calculated as

$$
P_{T}\left(t_{a b}\right)=\sum_{i=1}^{N\left(t_{a}\right)} \frac{1}{|T|^{2}} J\left(a, t_{a}^{i}\right)\left[\left.\frac{d t_{a b}\left(t_{a}\right)}{d t_{a}}\right|_{t_{a}=t_{a}^{i}}\right]^{-1}
$$

where $t_{a}^{i}$ represents the time of first crossing of the $x=a$ interface of the $N\left(t_{a b}\right)$ particles that have exactly the same transmission time $t_{a b}$. Moreover, this procedure can be easily extended to calculate the reflection and dwell time distributions. Until now, we have only considered transmitted particles which, as discussed above, are those with $Q\left(x_{0}\right)<|T|^{2}$, i.e., those that arrive at $x=a$ before $t_{1}$. Now, we can also consider those particles which are reflected, i.e., those that arrive at the $x=a$ interface later than $t_{1}$. According to the assumption that $J\left(b, t_{b}\right)$ is always positive, the reflected particles never cross the $x=b$ interface, and they remain within the barrier during time intervals enclosed by their first and last crossings of the $x=a$ interface. Contrary to the transmitted particles, the reflected ones cross this interface an even number of times, but the procedure to calculate their residence time in the barrier region $t_{a a}$ is analogous to that used to evaluate $t_{a b}$ [see Eq. (20)]. As for the calculation of the reflection time distribution, although the procedure is very similar, a relevant difference arises from the fact that not all the reflected particles enter into the barrier region, many of them being reflected without ever reaching $x=a$. The fraction of particles that enter the barrier and are ultimately reflected, $\left|R_{1}\right|^{2}$, can be directly obtained from $Q(a, t)$, since its absolute maximum is precisely $|T|^{2}+\left|R_{1}\right|^{2}$. The rest of the reflected particles, i.e., a fraction of the total equal to $|R|^{2}-\left|R_{1}\right|^{2}$, never enter the barrier and, as a consequence, have zero reflection time. Thus, the reflection time distribution must always include a term $\left[|R|^{2}-\left|R_{1}\right|^{2}\right] \delta(t)$ to preserve the normalization. The distribution of reflection times of those particles that actually enter the barrier region is obtained following a procedure analogous to that of Eq. (22) for transmitted particles, but now for those particles arrive at $x=a$ later than $t_{1}$. In this way, the reflection time distribution $P_{R}\left(t_{a a}\right)$ is obtained:

$$
\begin{aligned}
P_{R}\left(t_{a a}\right)= & \frac{|R|^{2}-\left|R_{1}\right|^{2}}{|R|^{2}} \delta(t) \\
& +\frac{1}{|R|^{2}} \sum_{i=1}^{N\left(t_{a a}\right)} J\left(a, t_{a}^{i}\right)\left[\left.\frac{d t_{a a}\left(t_{a}\right)}{d t_{a}}\right|_{t_{a}=t_{a}^{i}}\right]^{-1},
\end{aligned}
$$

where the $t_{a}^{i}$ represent the first time of arrival to $x=a$ of the $N\left(t_{a a}\right)$ reflected particles that have exactly the same residence time in the barrier. Finally, consistently weighting $P_{T}\left(t_{a b}\right)$ and $P_{R}\left(t_{a a}\right)$ by $|T|^{2}$ and $|R|^{2}$, respectively, we can directly obtain the dwell time distribution $P_{D}(t)$ :

$$
\begin{aligned}
P_{D}(t)= & {\left[|R|^{2}-\left|R_{1}\right|^{2}\right] \delta(t)+\sum_{i=1}^{N\left(t_{a b}\right)} J\left(a, t_{a}^{i}\right)\left[\left.\frac{d t_{a b}\left(t_{a}\right)}{d t_{a}}\right|_{t_{a}=t_{a}^{i}}\right]^{-1} } \\
& +\sum_{i=1}^{N\left(t_{a a}\right)} J\left(a, t_{a}^{i}\right)\left[\left.\frac{d t_{a a}\left(t_{a}\right)}{d t_{a}}\right|_{t_{a}=t_{a}^{i}}\right]^{-1}
\end{aligned}
$$

$t$ being equal to $t_{a b}$ or $t_{a a}$ for transmitted and reflected particles, respectively.

Notice that in all the considered cases, the distributions of tunneling times have been obtained without calculating trajectories. All that is needed is the time-evolved wave function, which is used to calculate the current probability density at the boundaries, and to link the corresponding times of arrival. Again, this represents a very important improvement in the efficiency of the numerical procedures because a very large number $\left(10^{4}-10^{5}\right)$ of trajectories is usually needed to obtain reliable distributions. Finally, it is worth remarking again that although the arrival time distributions are perfectly defined within the standard interpretation of $\mathrm{QM}$, the distributions of transmission and reflection times only make sense in Bohm's framework because a causal connection between the crossings of the two boundaries of the barrier is needed to calculate $t_{a b}$ and $t_{a a}$.

\section{INTUITIVE INTERPRETATION OF THE SCATTERING OF WAVE PACKETS}

Let us now consider the implications of the noncrossing property of Bohm trajectories on the intuitive interpretation of the scattering of wave packets by one-dimensional potential barriers. In this regard, Landauer and Martin [9] have made skeptical comments concerning two limit cases: (i) a wave function composed of two spatially separated packets incident on an opaque barrier; and (ii) a very long wave packet (a day in duration) incident on a very short but high barrier. In the first case they wrote "wave packets in succession, separated by a long time interval ... all the transmitted paths still come from the very first tip of the first packet," and in the second, "all the transmitted packet will come from about the first microsecond of the incident packet ... a day is long compared to any of the kinetic times associated with such a short barrier." The second example is difficult to analyze numerically for obvious reasons, but we will use an enriched version of the first one to show that Bohm's results are fully compatible with an intuitive interpretation of both cases. In particular, we consider the double-packet wave function, defined in Sec. III B [Eq. (21)], which evolves towards a double-barrier structure that has a resonance around $k_{1}$. Since $k_{1}$ is the center of the rear packet spectrum, this 


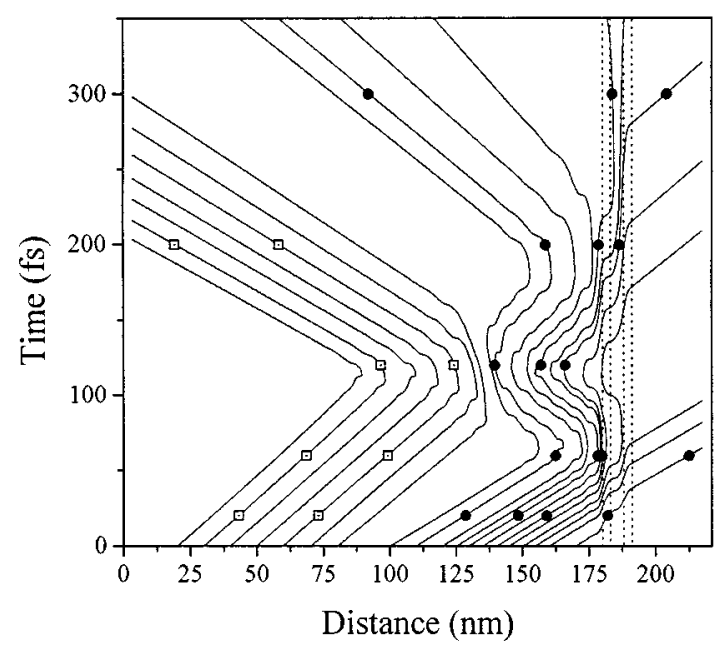

FIG. 6. Representative Bohm trajectories associated with double-packet scattering of Fig. 4. The position of the barriers is indicated by vertical dashed lines. The marks (squares for the rear packet and circles for the front one) are a visual aid to relate the trajectories with the evolution of the wave function shown in Fig. 7. Notice that the five horizontal lines (constant time) defined by the marks correspond to the five "snapshots" of the picture motion of the wave function shown in Fig. 7.

second packet is expected to practically control the whole transmission probability (this emphasizes the unexpected features of the first case considered by Landauer and Martin). Figure 6 shows selected Bohm trajectories and Fig. 7 is a picture motion of the traveling wave packet as obtained by numerical integration of the TDSE. At the beginning, the front packet travels faster towards the barrier and arrives to it

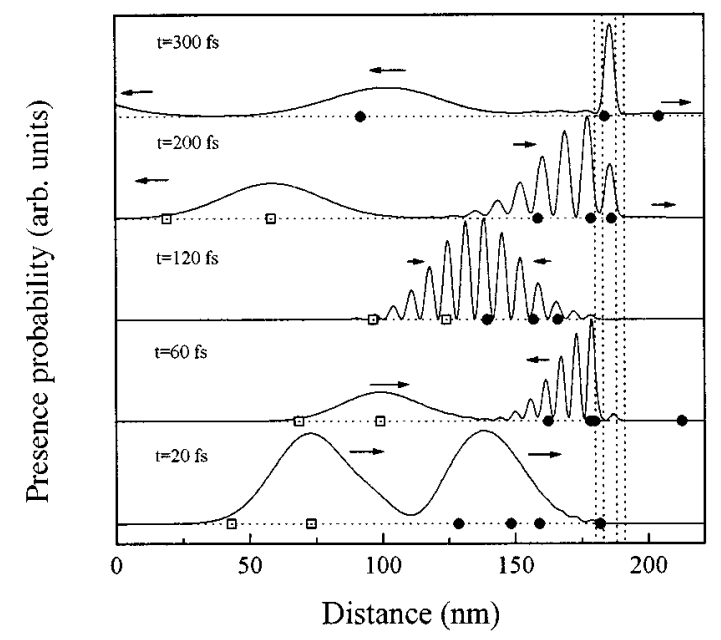

FIG. 7. Picture motion of the same double-packet wave function considered in Fig. 6, calculated by numerical integration of the time-dependent Schrödinger equation. Five representative "snapshots" obtained at different times are shown with the vertical scale arbitrarily changed in each case for clarity (although the norm of the wave function is always unity, it does not seem so because of the scale changes). The marks are visual aids that indicate the position of some related Bohm trajectories shown in Fig. 2. The double-barrier position is indicated by the vertical dashed lines, and the arrows indicate the sense of motion of the two packets.

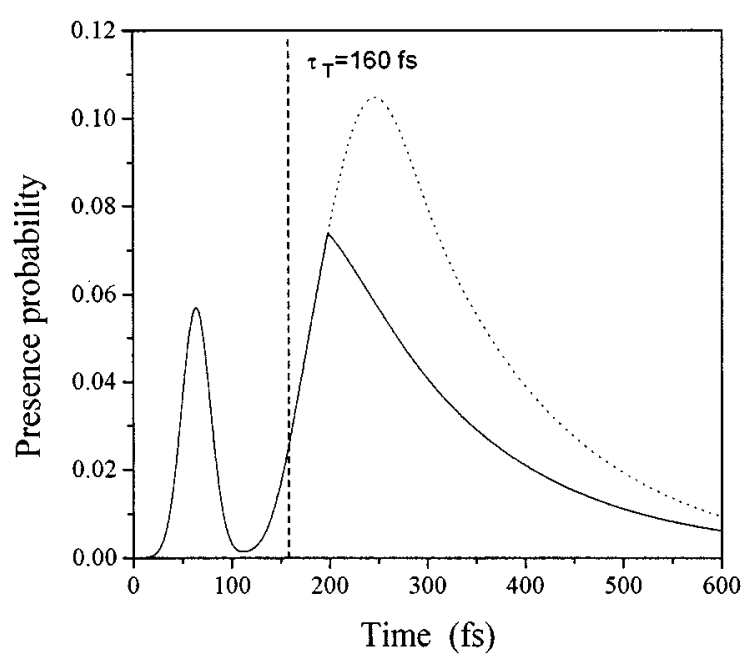

FIG. 8. Ensemble probability of finding the particles described by the double-packet wave function of Figs. 6 and 7 as a function of time during the scattering with the double-barrier potential. The dotted line corresponds to $D(t)$, which represents the probability of finding the particles in the barrier region, independently of the scattering channel (transmission or reflection). The continuous line corresponds to $D_{T}(t)$, which is the probability of finding to be transmitted particles in the barrier region. The vertical line indicates the average transmission dwell time as defined in expression (8), which in this case is $160 \mathrm{fs}$.

in first position. Only a small portion of this first packet is transmitted, and the rest of it is reflected with roughly the same momentum $\left(k_{2}\right)$. At times of the order of 120 fs strong interference effects take place between the two packets, which in Fig. 6 appear as a collision between Bohm trajectories. Momentum is interchanged, and the front packet travels again towards the barrier, but now with roughly $k_{1}$. This change of momentum can be noticed in Fig. 6 by a change of the slope of the trajectories, but could also be appreciated in the spatial oscillations of the real (or the imaginary) part of the wave function [30]. This second attempt to cross the barrier is more successful because now the front packet reaches the double barrier under resonance (accumulation of particles in the well is also apparent in both figures). In the Schrödinger picture, there is not an unambiguous criterion to decide whether the observed self-interference effects correspond to the scattering or to the crossing of wave packets. However, although both interpretations are equally acceptable, only the first one is compatible with Bohm's picture. The intuitive idea that the second wave packet, being resonant, controls most of the transmission continues to be valid. What Bohm's formulation tells us is that the second packet does its job by pushing the first one with the appropriate momentum towards the barrier, instead of being transmitted itself. A similar point of view, and the same example analyzed in Figs. 6 and 7, is also suitable to enlighten the case of the one-day-long wave packet impinging upon a very short and opaque barrier.

To establish a clear distinction between the transmission time [as defined in expression (8)] and the duration of the whole scattering process, we have plotted in Fig. 8 the probability of finding a particle in the barrier region $D(t)$, and that of finding a particle to be transmitted in the same spatial 
interval, $D_{T}(t)$. Two different peaks are perceived that correspond to the sequential interaction of the first wave packet with the barrier. In the first interaction event, $D_{T}(t)$ coincides with $D(t)$, and this means that all the particles that enter the barrier region are ultimately transmitted. The duration of the whole scattering process, which in this case is longer than $400 \mathrm{fs}$, can be appreciated to be much different from the average transmission time $\left(\tau_{T} \sim 160 \mathrm{fs}\right)$. The period of time between the successive interactions of the front packet with the barrier (separation of peaks in the figure) does not contribute to the transmission time, while it is certainly a relevant portion of the whole scattering process. This indirectly explains why the scattering of the one-day-long packet can be roughly interpreted as a steady-state situation (the common sense view according to Ref. [9]). What Bohm's formulation tells us is that most of the packet is reflected by self-interference effects and not by direct interaction with the barrier (it is reflected by the quantum potential in regions where the classical potential is zero). As a consequence, although the average transmission time can be of the order of picoseconds, the transmission of the small tip of the front packet can take much more time, i.e., as much as the interference time of the finally reflected packet (of the order of a day).

To finish with the intuitive interpretation of the wavepacket scattering, let us take another look at Fig. 6. In this figure we see that Bohm trajectories can be reflected for two different reasons: (i) interaction with the classical potential (i.e., the particles collide with the barrier) and (ii) collision with other trajectories traveling in the opposite direction. The second process is responsible for the reflection of those particles of the first packet, which never reach the barrier, and for the reflection of the entire second packet. These collisions between Bohm particles are related to the quantum potential in regions where the classical potential is zero but, for them to occur, there should be particles coming from right to left. In this regard, if the initial wave packet is prepared as a superposition of eigenstates incident from left to right (as is always assumed in scattering thought experiments), and the classical potential is zero for $x>b$, then finding particles coming from the right-hand side in this region will be at least very uncommon. This observation provides further intuitive support to the assumption of a positive current $J\left(b, t_{b}\right)$ or, equivalently, of the absence of reentrant trajectories at the $x=b$ boundary. From the above discussion, we conclude that the noncrossing property of Bohm trajectories does indeed allow an intuitive interpretation of the scattering of wave packets by potential barriers, and this means that claims that Bohm's approach provides counterintuitive results are only subjective appreciations.

\section{CONCLUSIONS}

Several aspects of the tunneling of electrons through potential barriers have been examined within the framework of Bohm's interpretation of quantum mechanics. In particular, we have focused our attention on the noncrossing property of the electron's trajectories. Although this property is also valid for three-dimensional problems, we have only considered the particular case of one-dimensional potentials and wave functions.

It has been shown that, due to the noncrossing property, the average tunneling times and the position of the Bohm particles can both be calculated without integrating and weighting trajectories. The consequences of this fact for the possible use of these trajectories for the extension of the Monte Carlo simulation technique to quantum-based devices have also been emphasized. On the other hand, a method to obtain the distributions of dwell, transmission, and reflection times without calculating trajectories has been presented. This method generalizes that of McKinnon and Leavens [17], which has been shown to have some limitations, but preserves the fundamental idea of connecting arrival time distributions. The presented method (hundreds of times faster than explicitly calculated $10^{4}$ Bohm trajectories), not only largely improves the numerical efficiency in the calculation of distribution of tunneling times, but also allows the assessment of sharp features as those of Fig. 5(b), which would be very difficult to appreciate by calculating trajectories.

Finally, the intuitive physical interpretation of the scattering of wave packets by potential barriers has been considered within Bohm's picture. The obtained results show that not only are the Bohm trajectories fully compatible with the common interpretation of scattering processes, but that they can enlighten some aspects that are less clear in the standard interpretation of QM. In any case, it must be highlighted that the Bohm trajectories exactly reproduce the time-dependent behavior of the wave function so that all the dynamical information is contained in the solution of the time-dependent Schrödinger equation. In other words, one cannot consider that the behavior of $\Psi(x, t)$ is intuitive, and that of the Bohm trajectories counterintuitive because both lead to the same observable results. Claims that Bohm trajectories lead to counterintuitive results are subjective.

\section{ACKNOWLEDGMENTS}

The authors are grateful to the Dirección General de Investigación Científica y Técnica for supporting this work under Project No. PB94-0720.
[1] M. Büttiker, Phys. Rev. B 27, 6178 (1983).

[2] D. Sokolovski and L. M. Baskin, Phys. Rev. A 36, 4604 (1987).

[3] C. R. Leavens, Solid State Commun. 74, 923 (1990).

[4] C. R. Leavens, Solid State Commun. 76, 253 (1990).

[5] C. R. Leavens and G. C. Aers, in Scanning Tunneling Microscopy III, edited by R. Wiesendanger and H.-J. Güntherrodt
(Springer, New York, 1993), p. 105.

[6] D. Sokolovski and J. N. L. Connor, Phys. Rev. A 47, 4677 (1993).

[7] V. S. Olkhovski and E. Recami, Phys. Rep. 214, 339 (1992).

[8] E. H. Hauge and J. A. St $\varnothing v n e n g$, Rev. Mod. Phys. 61, 917 (1989).

[9] R. Landauer and Th. Martin, Rev. Mod. Phys. 66, 217 (1994). 
[10] R. S. Dumont and T. L. Marchioro II, Phys. Rev. A 47, 85 (1993).

[11] S. Brouard, R. Sala, and J. G. Muga, Europhys. Lett. 22, 159 (1993).

[12] D. Bohm, Phys. Rev. 85, 166 (1952).

[13] P. R. Holland, The Quantum Theory of Motion (Cambridge University Press, Cambridge, 1993).

[14] D. Bohm and B. J. Hiley, The Undivided Universe: An Ontological Interpretation of Quantum Mechanics (Routledge, London, 1993).

[15] C. Pagonis, Nature 364, 398 (1993).

[16] C. Dewdney and B. J. Hiley, Found. Phys. 12, 27 (1982).

[17] W. R. McKinnon and C. R. Leavens, Phys. Rev. A 51, 2748 (1995).

[18] M. Hagmann, Solid State Commun. 86, 305 (1993).

[19] X. Oriols, F. Martín, and J. Suñé, Solid State Commun. 99, 123 (1996).

[20] C. R. Leavens, Found. Phys. 25, 229 (1995).
[21] R. E. Salvino and F. A. Buot, J. Appl. Phys. 72, 5975 (1992).

[22] A. Goldberg, H. M. Schey, and J. L. Schwartz, Am. J. Phys. 35, 177 (1967).

[23] M. O. Vasell, J. Lee, and H. F. Lockwood, J. Appl. Phys. 54, 5206 (1983).

[24] X. Oriols, J. Suñé, F. Martín, and X. Aymerich, J. Appl. Phys. 78, 2135 (1995).

[25] J. G. Muga, S. Brouard, and D. Macías, Ann. Phys. (N.Y.) 240, 351 (1995).

[26] J. G. Muga, S. Brouard, and R. Sala, Phys. Lett. A 167, 24 (1992).

[27] C. R. Leavens, Phys. Lett. A 178, 27 (1993).

[28] J. Suñé, X. Oriols, F. Martín, and X. Aymerich, Appl. Surf. Sci. (to be published).

[29] G. R. Allcock, Ann. Phys. (N.Y.) 53, 311 (1969).

[30] R. Landauer and Th. Martin, Solid State Commun. 84, 115 (1992). 